\title{
Fiebre de los humidificadores: una rara variante de neumonitis por hipersensibilidad
}

\author{
A. CAMARASA ESCRIG, E. CHINER VIVES, C. SENENT ESPAÑOL, J. N. SANCHO \\ CHUST, M. LLOMBART CANTÓ, A. L. ANDREU RODRÍGUEZ
}

Sección de Neumología. Hospital Universitario San Juan. Alicante

HUMIDIFIER FEVER: AN UNUSUAL VARIANT OF HIPERSENSIVITY PNEUMONITIS

\begin{abstract}
RESUMEN
Se presenta el caso de una paciente que presentó tres episodios de fiebre, infiltrados pulmonares, linfocitosis en lavado broncoalveolar y finalmente en la biopsia transbronquial mostró granulomas no caseificantes. Se estableció la relación entre la exposición al aparato de aire acondicionado de su oficina, siendo diagnosticada de neumonitis por hipersensibildad tipo fiebre de los humidificadores. Se señala la rareza de dicho cuadro, probablemente infradiagnosticado y se revisan los mecanismos patogénicos resaltando la necesidad de establecer un alto índice de sospecha.
\end{abstract}

PALABRAS CLAVE: Neumonitis por hipersensibilidad. Fiebre de los humidificadores. Alveolitis alérgica extrínseca. Pulmón de los humidificadores.

\begin{abstract}
A case of a patient suffering three episodes consistent in fever, lung infiltrates, lymphocitosis in bronchoalveolar lavage and non-caseificant granulomata in transbronchial biopsy is reported.

A relationship between exposition to the air conditioner in the office and the clinical picture was stablished, and the patient was finally diagnosed from humidifiers fever. We enfatize the manifestations of this uncommon disease, probably infradiagnosed, and the patogenic mechanisms are reviewed.
\end{abstract}

KEY WORDS: Hipersensitivity pneumonitis. Humidifier fever. Extrinsec allergic alveolitis. Lung humidifier.

Camarasa Escrig A, Chiner Vives E, Senent Español C, Sancho Chust JN, Llombart Cantó M, Andreu Rodríguez AL. Fiebre de los humidificadores: una rara variante de neumonitis por hipersensibilidad. An Med Interna (Madrid) 2007; 24: 490-493.

\section{INTRODUCCIÓN}

La Neumonitis por hipersensibilidad, también llamada Alveolitis alérgica extrínseca, es una enfermedad de base inmunológica producida por gran variedad de antígenos que llegan al pulmón por vía inhalada, vehiculizados por polvos orgánicos, en ocasiones inorgánicos, de origen casi siempre ocupacional. Las dos variedades mejor conocidas a nivel mundial son el pulmón del granjero y el pulmón del cuidador de aves (1).

La aparición de neumonitis por hipersensibilidad en relación con sistemas de aire acondicionado contaminados en trabajadores de oficinas, también denominada Fiebre de los humidificadores, es de reciente descripción y puede pasar desapercibida si no existe un alto índice de sospecha clínica (2).

Presentamos el caso de una paciente afecta de episodios recidivantes de Fiebre de los humidificadores, en nuestro conocimiento el primer caso publicado en la literatura española.

\section{CASO APORTADO}

Mujer de 38 años fumadora de 21 años/paquete, diabética tipo II y con antecedentes de asma bronquial desde hacía 4 años sin ningún tipo de tratamiento ni seguimiento. Trabajaba como administrativa en una clínica dental desde hacía nueve meses, y coincidiendo con el cambio de trabajo y según refería en relación al mismo, venía presentando fiebre intermitente, sibilancias diurnas y nocturnas y disnea progresiva. Los síntomas habían empeorado en las últimas semanas a pesar de tratamiento antitérmico, por lo que acude a Urgencias en Julio de 2002, ingresando posteriormente.

A su ingreso presentaba regular estado general, fiebre de 38 ${ }^{\circ} \mathrm{C}, 20$ vpm, 65 ppm, buena hidratación y coloración. La auscultación cardiaca era normal y a la auscultación pulmonar destacaba una disminución del murmullo vesicular, con sibilancias espiratorias bilaterales. El resto de exploración no mostraba hallazgos de interés.

La radiografía de tórax al ingreso mostró patrón alveolointersticial bilateral parcheado de predominio perihiliar con alguna atelectasia laminar (Fig. 1). La gasometria arterial respirando aire ambiente

Trabajo aceptado: 15 de mayo de 2007

Correspondencia: Ana Camarasa Escrig. Sección de Neumología. Hospital Universitario San Juan de Alicante. Crta. Alicante-Valencia. 03550 San Juan. Alicante.e-mail: anacamarasa@hotmail.com 


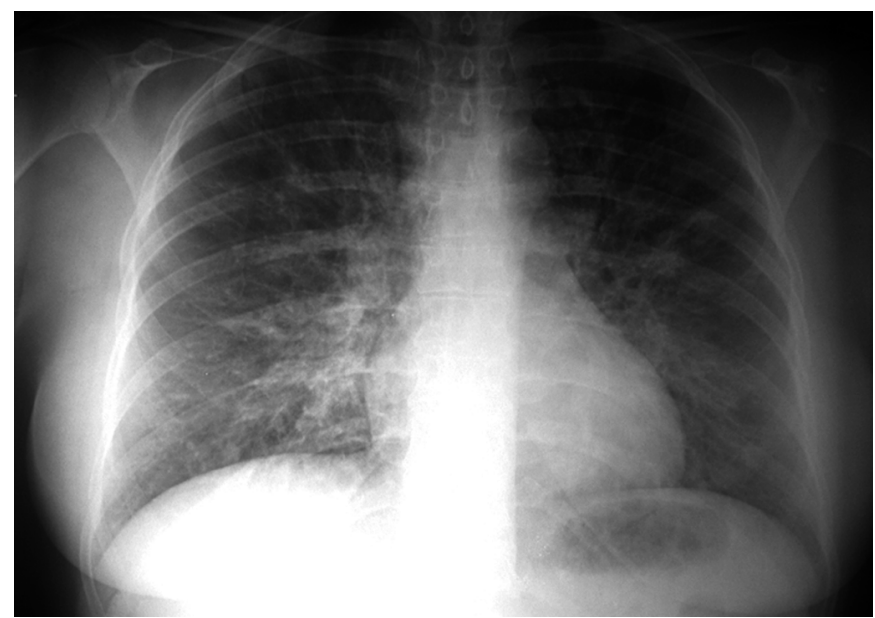

Fig. 1. Radiografía PA de tórax que muestra patrón alveolo-intersticial bilateral.

mostró: pH 7,45, $\mathrm{PO}_{2} 54,7 \mathrm{mmHg}, \mathrm{PCO}_{2} 35,6 \mathrm{mmHg}, \mathrm{HCO}_{3}$ 24,3 $\mathrm{mmol} / 1$ y $\mathrm{SaO}_{2} 89 \%$. En la analítica destacaba 15700 leucocitos con 90\% de neutrófilos y $0 \%$ eosinófilos, así como VSG $89 \mathrm{~mm}$. La bioquímica mostró glucosa de $198 \mathrm{mg} / \mathrm{dl}$ siendo el resto de parámetros normales a excepción de PCR de 17,29 mg/dl.

Se realizó TACAR de tórax que mostró patrón en vidrio deslustrado parcheado bilateral de predominio en lóbulos superiores y una pequeña área de consolidación atelectasia en lóbulo medio y língula; no había hallazgos de significación a nivel mediastínico.

Las pruebas de función respiratoria mostraron: FVC $2280 \mathrm{ml}$ (71\%), FEV1 $1770 \mathrm{ml}$ (64\%), FEV1/FVC 78 (90\%), DLCO 15,6 $\mathrm{ml} / \mathrm{min} / \mathrm{mmHg}(61 \%)$, DLCO/VA 4,69 (88\%), todo ello compatible con leve alteración restrictiva con moderada afectación de la difusión, corregida a volumen alveolar.

Se practicó fibrobroncoscopia el cuarto día de ingreso, no apreciando alteraciones en la vía aérea. El lavado broncoalveolar (BAL) mostró 500 celulas $/ \mu 1$, con $65 \%$ macrófagos, $20 \%$ linfocitos, $10 \%$ polimorfonucleares, 5\% eosinófilos con subpoblaciones linfoides CD4+ 46,4\% y CD8+ 29,9\%. La microbiología del BAL, incluyendo bacterias, Zhiel-Nielsen y cultivo de Lowenstein, así como hongos y parásitos fue negativa. El Zhiel y Lowenstein del broncoaspirado (BAS) fueron igualmente negativos, así como fueron negativos para malignidad la citología del BAL y del BAS. No se realizó biopsia transbronquial (BTB) al tener que interrumpirse la técnica por tos incoercible de la paciente. A su ingreso se había instaurado tratamiento con oxigenoterapia, amoxicilina-clavulánico $1 \mathrm{gr} / \mathrm{IV} / 8 \mathrm{hs}$, quedando apirética a las 24 horas y el $5^{\circ}$ día de ingreso se apreció una notable mejoría clínica y radiológica.

La IgE fue de 327,3 UI/ml y se solicitó RAST que se recibió posteriormente, siendo positivo para gramíneas, polen de olivo y epitelio de gato, negativo para ácaros y hongos. Las precipitinas séricas para Alternaria Tenuis, Aspergillus, proteínas aviares y Thermoactinomyces fueron asimismo negativas.

Ante la presencia de linfocitosis en el BAL y la rápida mejoría radiológica, se interrogó de nuevo a la paciente, refiriendo que los síntomas respiratorios se habían iniciado al cambiar de trabajo, encontrándose peor en la oficina, en su puesto de trabajo, donde tenía encima un aparato de aire acondicionado, mejorando durante el fin de semana y en vacaciones, reiniciando febrícula y disnea al reincorporarse a su jornada laboral.

El sexto día de ingreso hospitalario se agregó prednisona 40 $\mathrm{mg}$ /día y fue dada de alta a los 10 días de su ingreso con pauta descendente.

En un control posterior, un mes más tarde, la paciente estaba asintomática mostrando la exploración funcional respiratoria FVC 3.380 $\mathrm{ml}$ (105\%), FEV1 $2670 \mathrm{ml}$ (96\%), FEV1/FVC 79 (96\%), DLCO 19,8 $\mathrm{ml} / / \mathrm{min} / \mathrm{mmHg}(78 \%)$, DLCO/VA 4,68 (88\%) y sin evidencia de lesiones parenquimatosas en TACAR, reincorporándose a su trabajo sin problemas, tras haber practicado limpieza exhaustiva del aparato de aire acondicionado y de los sistemas de refrigeración.

Nuestra paciente no acudió a las sucesivas citas, aduciendo que se encontraba asintomática, hasta que en Enero de 2006 ingresa de nuevo por cuadro de malestar general, artromialgias, escalofríos, sin objetivarse fiebre hasta el momento del ingreso; no refería tos ni expectoración y había iniciado tratamiento con Azitromicina sin encontrar mejoría. Desde hacía tres meses trabajaba como administrativa y vendedora en un almacén de gres. Los síntomas se habían iniciado al poner en marcha los sistemas de calefacción por aire caliente en su oficina.

A la auscultación presentaba crepitantes bilaterales, así como un patrón intersticial micronodular bilateral en la radiografía de tórax. El TACAR de tórax evidenció opacidades en vidrio deslustrado, parcheadas, de predominio en lóbulos superiores con engrosamiento de septos interlobulillares (Fig. 2). En la analítica desta-

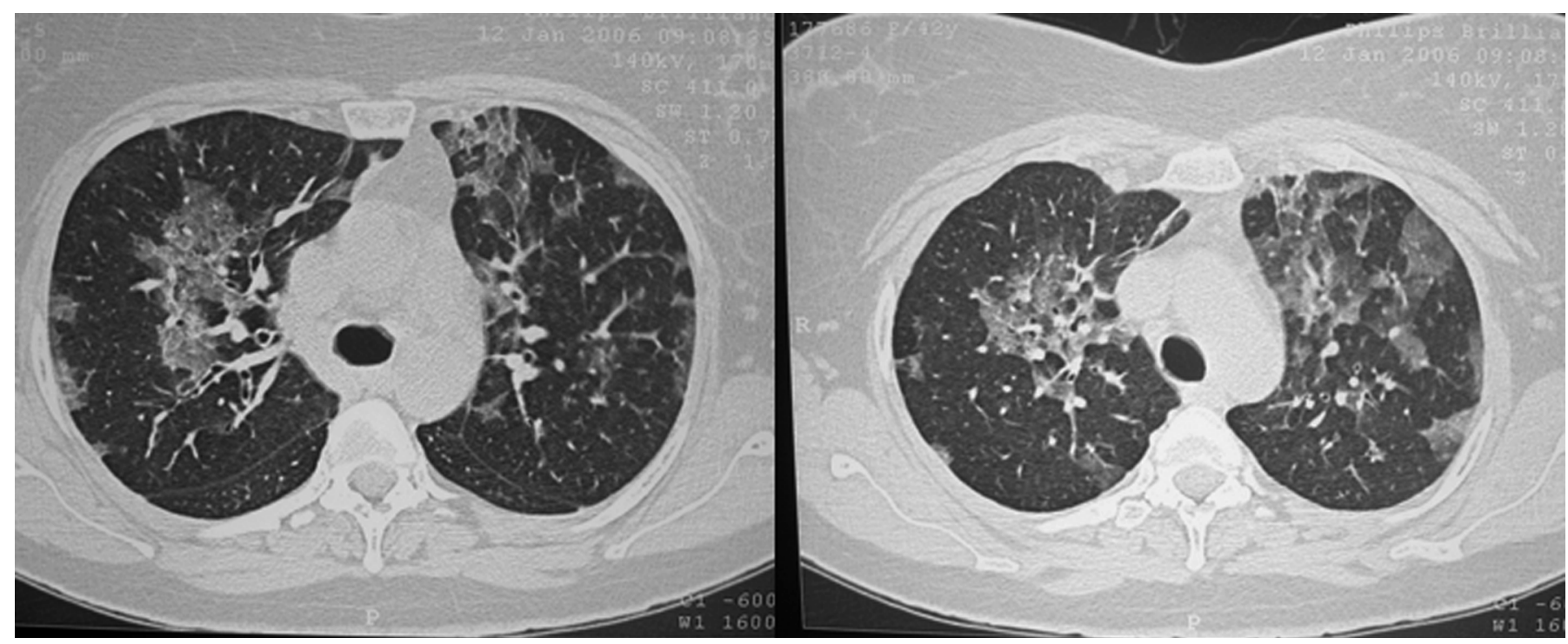

Fig. 2. TACAR de tórax que muestra opacidades en vidrio deslustrado, parcheadas, de predominio en lóbulos superiores con engrosamiento de septos interlobulillares. 
caba aumento de VSG, Ig E y PCR así como insuficiencia respiratoria aguda parcial $\mathrm{pH} 7,51, \mathrm{PO}_{2} 49,7 \mathrm{mmHg}, \mathrm{PCO}_{2} 35,9 \mathrm{mmHg}$, $\mathrm{HCO}_{3} 28,3 \mathrm{~mm} / 1$ y $\mathrm{SaO}_{2} 88,2 \%$.

Se practicó nueva fibrobroncoscopia con BAL que objetivó 357 celulas $/ \mu 1$, con $70 \%$ macrófagos, $20 \%$ linfocitos, $6 \%$ de polimorfonucleares, $4 \%$ basófilos, $1 \%$ eosinófilos con subpoblaciones linfoides CD4+ 45\% y CD8+37\%; no hubo crecimiento bacteriano, el Zhiel y Lowenstein fueron negativos y se practicó BTB donde se apreciaron áreas de alveolitis y bronquiolitis cerca de bronquios terminales con presencia de granulomas sin necrosis central.

Las pruebas de función respiratoria mostraron: FVC $2180 \mathrm{ml}$ (62\%), FEV1 $1590 \mathrm{ml}$ (57\%), FEV1/FVC 73 (92\%) DLCO 17 $\mathrm{ml} / \mathrm{min} / \mathrm{mmHg}$ (69\%), DLCO/VA 4,97 (96\%) compatible con alteración restrictiva con afectación de la difusión que mejoraron tras iniciar tratamiento con broncodilatadores y corticoesteroides en pauta descendente durante 30 días al igual que el ingreso previo.

\section{DISCUSIÓN}

En 1970 Banaszak y cols describieron por primera vez la aparición de neumonitis por hipersensibilidad en relación con sistemas de aire acondicionado contaminados en trabajadores de una oficina (2). Años más tarde Robertson y cols. mostraron un caso de fiebre de humidificadores donde el antígeno causal no eran Actinomicetos termofílicos (frecuentemente aislados en el agua caliente de los humidificadores), siendo el origen antigénico humidificadores de agua fría, lo que sugirió que el antígeno causante del cuadro podía depender del tipo de humidificador (3).

Debido a las características de la enfermedad, para establecer un diagnóstico correcto es importante considerarla ante síntomas respiratorios intermitentes con afectación sistémica, con una evolución recidivante o progresiva, acompañada de patrón intersticial en la radiografía. A continuación, es necesario buscar una asociación entre la presentación de los síntomas y la exposición al antígeno, conociendo las principales fuentes antigénicas; no obstante, la presencia de precipitinas específicas no indica enfermedad activa, sólo contacto previo con el antígeno, por lo que son altamente sensibles pero poco específicas $(4,5)$. De hecho, la estrategia diagnóstica en la neumonitis por hipersensibilidad depende de la probabilidad inicial de presentarla (6).

El BAL es de gran utilidad para orientar el cuadro, puesto que un BAL normal excluye esta entidad, pero en ningún caso es por sí mismo diagnóstico. El perfil citológico característico consiste en un aumento del recuento total de células y un incremento de linfocitos, a expensas fundamentalmente de CD8+, con inversión del cociente CD4+/CD8+. Sin embargo, como en nuestro caso, algunos trabajos han mostrado predominio de linfocitos CD4+ en pacientes diagnosticados de pulmón de granjero, con incremento del cociente (1). Asimismo, también se han descrito casos con descenso de dicho cociente en la llamada neumonitis por hipersensibilidad tipo verano, (característica en Japón) (7), con lo que podemos concluir que el fenotipo linfocítico del BAL es variable según el tipo y grado de exposición antigénica (8).

De hecho, en el pulmón de humidificadores se ha descrito incremento en CD4+ con cociente mayor que el visto para neumonitis tipo verano y menor que para el pulmón del granjero. La razón para estas diferencias no está del todo aclarada, pero es posible que el fenotipo linfocítico dependa de varios factores, como la naturaleza o dosis de antígeno, la duración de la exposición o el estadio de la enfermedad $(5,8)$. En este sentido, la neumonitis tipo verano y pulmón del cuidador de aves parecen estar causadas por una exposición continua a bajas dosis de antígenos, mientras que el pulmón de humidificadores es debido a una exposición a altas dosis de antígeno de forma intermitente.

La neumonitis por hipersensibilidad representa en la actualidad un problema a la hora de abordar el diagnóstico. Por ello se han establecido una serie de criterios, revisados en 1997. Los criterios mayores incluyen: síntomas compatibles, exposición antigénica apropiada, hallazgos radiológicos, linfocitosis en el BAL, cambios patológicos compatibles y provocación bronquial específica positiva y los criterios menores: crepitantes en bases, alteración de la difusión, hipoxemia arterial en reposo o tras ejercicio, precisando 4 y 2 respectivamente para establecerlo (9). En éstos se incluye la presencia de linfocitosis sin especificar el fenotipo linfocitario. Además, en un estudio posterior Lacasse y cols (6) proponen una serie de criterios clínicos predictivos: exposición a agente causal conocido, episodios recurrentes de síntomas, clínica tras 4-8 horas de la exposición, pérdida de peso, crepitantes y precipitinas en suero, destacando el hecho que no incluya el lavado broncoalveolar o biopsia, determinándose un valor predictivo positivo de un $98 \%$ si están presentes los seis criterios.

En la mayoría de los casos en los que se llega a precisar un diagnóstico anatomopatológico, cuando el resto de pruebas son discordantes o hay dudas, éste puede obtenerse mediante BTB. Los hallazgos más frecuentemente son: granulomas no caseificantes mal definidos y de pequeño tamaño cercanos a los bronquiolos terminales, constituidos por agregados de macrófagos y células gigantes multinucleadas; infiltrados parcheados mononucleares en las paredes alveolares con distribución broncocéntrica; histiocitos grandes con citoplasma espumoso en los alveolos y el intersticio, aunque no siempre se identifican lesiones características en la biopsia pulmonar, no siendo por tanto imprescindible para establecer el diagnóstico (10).

Concluimos que no existe ninguna prueba que sea patognomónica de neumonitis por hipersensibilidad, ni siquiera BAL o la BTB. Un manejo adecuado de las diferentes técnicas disponibles (precipitinas, radiología, estudio funcional respiratorio, lavado broncoalveolar y BTB) en el contexto de una historia clínica exhaustiva y compatible, puede confirmar nuestra sospecha inicial de Neumonitis por hipersensibilidad. 


\section{Bibliografía}

1. Ando M, Suga M. Hypersensitivity pneumonitis. Curr Opin Pulm Med 1997; 3: 391-5

2. Banaszak EF, Thiede WH, Fink JN. Hypersensitivity pneumonitis due to contamination of an air conditioner. N Eng J Med 1970; 283: 271-6.

3. Robertson AS, Burge PS, Wieland GA,Carmalt MH. Extrinsec allergic alveolitis caused by a cold water humidifier. Thorax 1987; 42: 32-7.

4. Baur X, Richter G, Pethran A, Czuppon AB, Schwaiblmair M. Increased prevalence ogf IgG-induced sensitzation and hypersensitivity pneumonitis (humidifier lung) in nonsmokers exposed to aerosols of a contaminated air conditioner. Respiration 1992; 59: 211-4.

5. Suda T, Sato A, Ida M, Gema H, Hayakawa H, Chida K. Hypersensitivity pneumonitis associated with home ultrasonic humidifiers. Chest 1995; 107: 711-7.

6. Lacasse Y, Selman M, Costabel U, Dalphin J, Ando M, et al. Clinical diagnosis of Hypersensitivity pneumonitis. Am J Respir Crit Care Med 2003; 168: 952-8.

7. Sugita T, Ikeda R, Nishikawa A. Analysis of Trichosporon isolates obtained from the houses of patients with summer-type hypersensitivity pneumonitis. J Clin Microbiol 2004; 42: 5467-71.

8. Ando M, Konishi K, Yoneda R Tamura M. Difference in the phenotypes of bronchoalveolar lavage lymphocytes in patients with summertype hypersensitivity pneumonitis, farmer's lung, ventilation pneumonitis, and bird fancier's lung: report of a nationwide epidemiologic study in Japan. J Allergy Clin Immunol 1991; 87: 1002-9.

9. Schuyler M, Cormier Y. The diagnosis of hypersensitivity pneumonitis. Chest 1997; 111: 534-6.

10. Lacasse Y, Fraser RS, Fournier M, Cormier Y. Diagnostic accuracy of transbronchial biopsy in acute farmer's lung disease. Chest 1997; 112: 1459-65. 